\title{
Interactive Simulation of Fibrin Fibers in Virtual Environments
}

\author{
Jeffrey L. Schoner ${ }^{1}$, Michael R. Falvo ${ }^{2,3}$, Susan T. Lord ${ }^{4}$, Russell M. Taylor $I^{1,2,3}$ and Ming C. Lin ${ }^{1}$ \\ University of North Carolina at Chapel Hill
}

\begin{abstract}
We present a data-driven method for interactive simulation and visualization of fibrin fibers, a major component of blood clotting. A fibrin fiber is a complex system consisting of a hierarchy with at least three separate levels of detail. Using measurements acquired with an atomic force microscope (AFM) at the smallest scale in this hierarchy, a physically-based model for the larger scales can be constructed and then simulated. Unlike most traditional work dealing with Monte Carlo (MC) or Molecular Dynamics (MD) simulations, our method makes simplifying assumptions about the simulation and enables interactive visualization of simulated fibers in a virtual environment.
\end{abstract}

CR Categories: I.3.5 [Computer Graphics]: Computational Geometry and Object Modeling-Physically based modeling; J.3 [Life and Medical Sciences]

Keywords: physically-based simulation, medicine

\section{INTRODUCTION}

When blood vessels are cut or injured, the loss of blood from the system is prevented by the transformation of fluid blood into a solid blood clot. This process is called coagulation or clotting. A blood clot consists of a plug of platelets enmeshed in a network of insoluble fibrin fibers. Since fibrin fibers are the major structural component of blood clots, determining the mechanical properties of these fibers can provide new insights into the wound healing process and advance our understanding of heart attacks and strokes. There has been much interest in determining the response of fibrin clots to mechanical stresses. Past investigations have mainly focused on the bulk mechanical properties of whole fibrin clots [10, 11, 13, 14, 16, 19, 20, 27, 28, 29, 36, 38, 39, 42, 44, 43, 48, 52]. There remains much to be discovered and understood about the properties of the individual fibers that comprise the clot. In fact, to our knowledge, the only work studying the mechanical properties of individual fibrin fibers is a recently published analysis based on similar experimental data [15]. However, none of this work has been concerned with the construction and simulation of more detailed structural models for individual fibers.

At the University of North Carolina (UNC) at Chapel Hill, we have developed a virtual reality interface for manipulating nanostructures, called the nanoManipulator [18, 47] as shown in Figure 1 Our nanoscale science research group has been developing suitable protocols to perform measurements of mechanical properties on individual fibrin strands. The nanoManipulator VR interface is used to locate the desired modification site and its polyline virtual

\footnotetext{
${ }^{1}$ Department of Computer Science, Sitterson Hall, UNC-CH, Chapel Hill, NC 27599-3175, USA, \{jschoner,taylorr,lin\}@ @s.unc.edu

${ }^{2}$ Deparment of Physics and Astronomy, falvo@ physics.unc.edu

${ }^{3}$ Curriculum in Applied and Materials Sciences

${ }^{4}$ Deparment of Pathology and Laboratory Medicine, stl@med.unc.edu
}

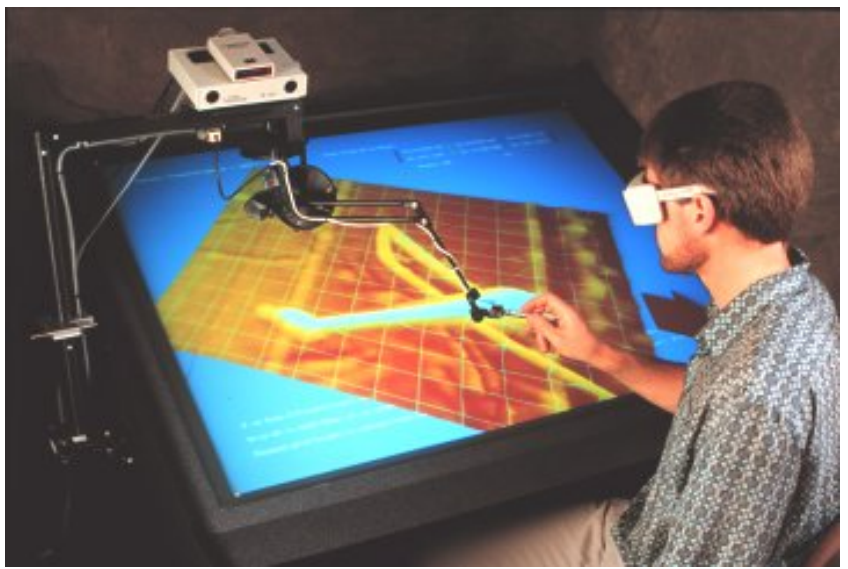

Figure 1: UNC nanoManipulator system: A virtual reality interface for manipulating nano-structures.

tip is used to effect the modification. We also use this instrumentation to measure the rupture force and other mechanical properties of individual fibrin fibers.

Our ultimate goal is to use nanoManipulator to perform various virtual experiments. By simulating and predicting how the network of fibers may behave under different conditions in a "virtual laboratory", we can interactively change the mechanical properties of individual fibrin fibers due to various factors. Assuming that a computational model for describing the mechanical properties of individual fibrin fibers is given, we can validate it by comparing the simulation results from virtual experiements against the measurements taken in the laboratory under varying conditions. Given a validated model for individual fibers, we believe that it is feasible to develop a virtual prototyping system for predicting the group behavior of blood clots. This approach can potentially lead to the development of new wound dressing methods.

However, simulating individual fibers is a rather difficult task, as there are no validated models. One possibility is to use molecular dynamics to accurately compute the mutual interaction among fibrin molecules and with the solvent molecules. However, such simulations involve highly complex and costly computations at extremely small time steps and could easily take weeks or months even on high-performance parallel computers - clearly unsuitable for interactive manipulation in a virtual environment. Predicting the behavior of the entire blood clot using such techniques will be even more daunting as there are numerous fibers in each blood clot and simulating their mutual interaction will be computationally intractable. As a result, modeling and simulating fibrin fibers remains a major computational challenge for real-time manipulation in a virtual environment.

Main Results: In this paper, we show that building upon the experimental data, it is possible to derive a computational model for individual fibrin fibers by a combination of data-driven modeling and physically-based simulation. More specifically, each fibrin fiber is a complex system consisting of a hierarchy with at least three levels of detail. Using measurements acquired with a VR interface at the smallest scale in this hierarchy, we construct a physically-based 
model for the medium level protofibril, which can then be simulated as a group to predict the behavior of each individual fibrin fiber in a virtual laboratory. Unlike Monte Carlo (MC) or Molecular Dynamics (MD) simulations, our approach makes appropriate simplifying assumptions, enabling us to achieve interactive simulation of individual fibrin fibers in a virtual environment.

Organization: The rest of the paper is organized as follows. Section 2 describes previous work in related areas. We provide a brief overview of our approach and the simulation framework hierarchy in Section 3. Section 4 details various steps used in our simulation, as well as our methods for visualizing it. We discuss the implementation issues and demonstrate our prototype system in Section 5 . We conclude with future research directions and open research issues in this area.

\section{RELATED WORK}

There exist no known, validated computational models to represent individual fibrin fibers. To our knowledge, the model we present in this paper is the first solution to this rather challenging problem. Our approach is inspired by recent work on data driven modeling in computer graphics and built upon existing constraint solving techniques.

\subsection{Data-Driven Modeling}

Traditionally, computational models based on developed analytical models have been used to design efficient numerical methods and effective algorithms for modeling various elements and phenomena in computer graphics. However, most existing analytical models were first derived based on observations of physical experiments under various simplifying assumptions. These first-principle models are not always able to capture higher order effects.

With the availability of high-quality sensors and measurement instruments, the new data gathered by these devices have been found to be sufficient to represent systems described by existing known analytical models. In addition, many objects, structures and effects currently do not have sufficiently rich model representations. Therefore, new approaches that attempt to process, retarget, refit, and adapt captured data to transform known measurements into images and animations have been proposed in many areas. They include shape representation [37 1], rendering [8], surface reflectance [24 26], character animation [46], simulation of deformable objects [23, 32, 22, 40], medical applications [25, 7, 49, 5, 17], etc.

\subsection{Constraint Enforcement}

A dynamical system is often subject to various internal and external constraints. To model and simulate individual fibrin fibers, various constraints and chemical bonds must be taken into account.

Constraint solving has been extensively studied in many different fields, such as CAD/CAM, molecular modeling, and theorem proving [4, 6, 21]. Some of the common approaches include numerical algebraic techniques, graph-based algorithms, logical interference and term rewriting, symbolic algebraic solvers, and propagation methods [4].

One of the simplest and commonly used techniques is penalty methods for enforcing constraints. They proceed to cancel the applied forces that break the constraints through restoring forces. While effective for certain situations, our problem requires more exact constraint enforcement. We adopt a technique called constrained dynamics proposed by Witkin [53] in physically-based modeling, which we will describe in more detail in Section 4

\section{FIBRIN}

Fibrin, as a substance involved in blood clotting, consists of four distinct levels of detail [51], from largest to smallest:

Fiber Webs Webs of fibers serve as a scaffold for a blood clot, which includes an aggregation of platelets. These webs consist of large numbers of fibrin fibers and come into existence when branching occurs during the formation of structures at smaller levels. A photograph of such a web can be seen in Figure2

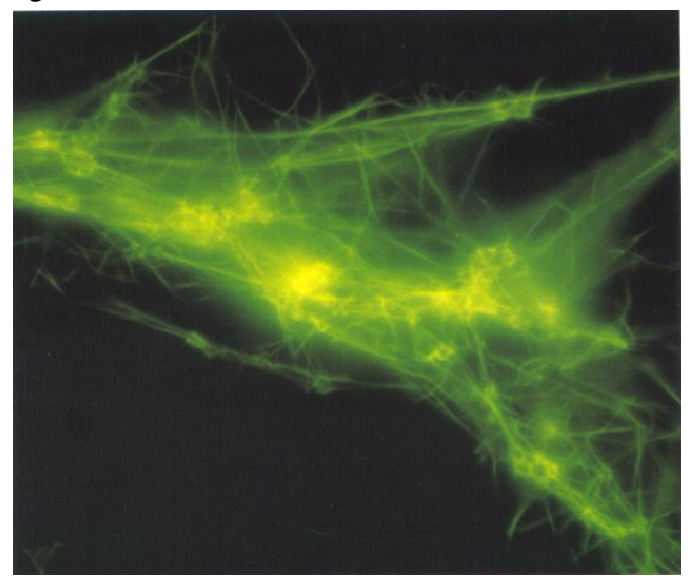

Figure 2: Photograph of a web of fluorescent fibrin fibers.

Fibrin Fiber Thick fibrin fibers are composed of many thin fibrin fibers wrapped around one another. Each thin fibrin fiber is composed of several protofibrils, which have aggregated in an organized manner. The number of protofibrils present can vary widely, with thin ones containing as few as 10 and thick ones hundreds.

Protofibril A protofibril is a double-chained structure, consisting of fibrin monomers. Initially the monomers are held together by non-covalent forces, i.e. van der Waals forces, but subsequently covalent bonds are added linking the end domains between adjacent monomers. Figure 3 shows the geometry of how the monomers combine to form a protofibril chain. The number of monomers present in a protofibril is typically around 20, although there is substantial variation dependent on the experimental conditions.

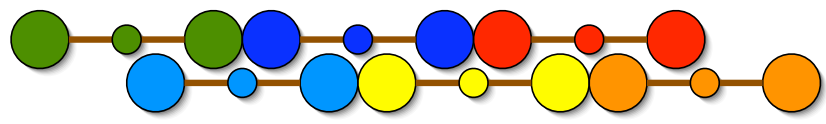

Figure 3: Protofibril structure. Each protofibril consists of two bonded chains of fibrin monomers. The components of separate fibrin monomers are shown in unique colors.

Fibrin Monomer The thrombin enzyme converts a fibrinogen molecule is converted into fibrin monomer. The process in volves the cleaving of certain bonds, which allows fibrin monomer to polymerize into protofibrils. Individual fibrinogen molecules are large, complex chains consisting of approximately 25,000 atoms.

At a coarser scale, the monomer can be divided into three important features: two large end domains and a smaller central domain. As force is applied to move the ends away from each other they unravel. In Figure 4 the simplified structure of the monomer is shown with these three features as well as lines in between them showing the current length of the unraveled portion of the chain. 


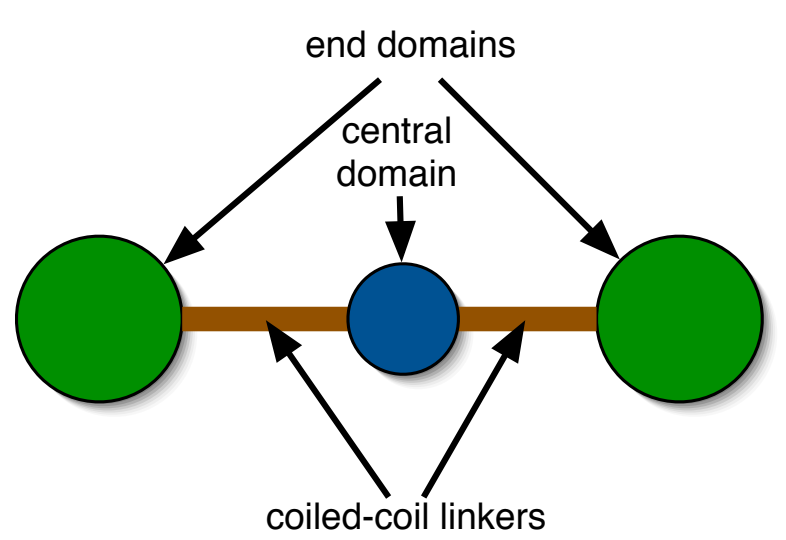

Figure 4: Simplified fibrin monomer structure. The large chain can be split into 2 end domains, one central domain and 2 connecting coiled-coil linkers.

This paper focuses on simulation of fibrin fibers using measurement data acquired at the lowest level.

\subsection{Measurement of Fibrinogen Properties Using the nanoManipulator VR Interface}

The experimental data for the fibrin experiments was obtained using our nanoManipulator virtual-environment interface to a scannedprobe microscope. Fluorescent data captured on a scientific video camera was displayed aligned with motion of the probe tip, enabling us to watch the deformation of the fibers while recording force measurements. Initial placement of the probe was done by hand in 3D; semi-automatic controls were used to move the tip along desired trajectories during deformation.

The nanoManipulator VR interface plays a crucial role in performing scientific experiments otherwise not possible. This VR interface is used to locate the desired modification site, and its polyline virtual tip is used to effect the modification. It also measures the rupture force and other mechanical properties of individual fibrin fibers. Fig. 5 shows an example of force measurements taken by the nanoManipulator. Later on, we plan to use the nanoManipulator to perform virtual experiements on simulated fibrin fibers under varying conditions.

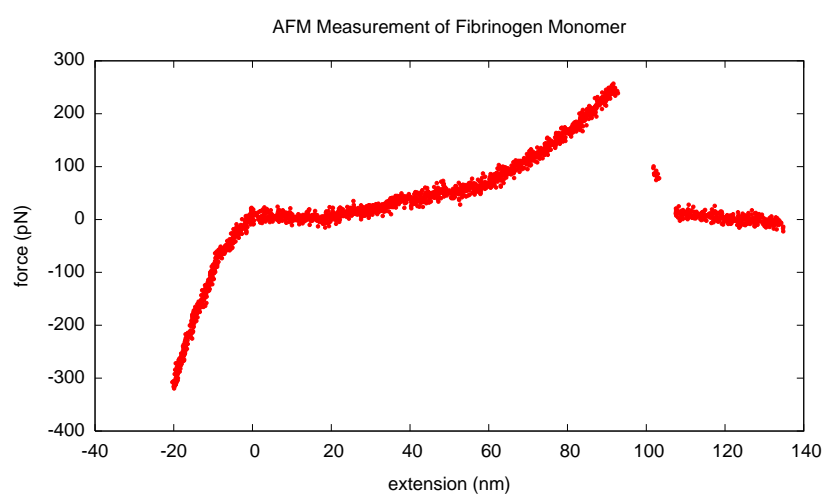

Figure 5: A measurement taken of an individual fibrinogen monomer using AFM.

\subsection{Fitting Models}

To capture the physical behavior collected in the above described measurements, it is necessary to fit them to a mathematical model.
Two models have been investigated in our work: Hookean and wormlike chain (WLC). The Hookean model is a common linear spring, whose behavior is described by

$$
f_{\text {Hooke }}=-k_{s}(x-l),
$$

where $f_{\text {Hooke }}$ is the force exerted at the ends of the monomer, $x$ is the elongation of the monomer, $l$ is the rest length and $k_{s}$ is the stiffness. When using the Hookean model, $k_{s}$ and $l$ are fitted from the measurements.

The WLC model also relates force and extension length, but better estimates the non-linear extension behavior of the fibrin monomer. It has been used in modeling other polymeric structures such as titin [35]. This model is governed by,

$$
f_{\mathrm{WLC}}=-\frac{k T}{4 b}\left(\left(1-\frac{x}{L}\right)^{-2}-1+\frac{4 x}{L}\right),
$$

where $f_{\mathrm{WLC}}$ and $x$ are analogous to those in the Hookean model. Both, the persistence length, $b$, which is analogous to bending rigidity and the contour length, $L$, the maximum attainable extension of the molecule are specific to a given molecular structure. $T$ is the temperature in degrees Kelvin at which the experiment is conducted (typically, 293K). $k$ is the Boltzmann constant $\left(1.38 \times 10^{-23} \mathrm{~m}^{2} \mathrm{~kg}\right.$ $\left.\mathrm{s}^{-2} \mathrm{~K}^{-1}\right)$.

When using the WLC model, $L$ and $b$ must be fitted.

\subsection{Fitting Process}

The data acquired from the AFM contains noticeable noise (see Figure 5), although for AFM measurements they are fairly low-noise. Additionally, the data has been collected over the entire course of the experiment (from pressing down, through elongation and past detachment), while we are only interested the elongation stage.

A two step process is carried out to fit the data. First, the data is cropped to the elongation portion. This involves ignoring measurements with an extension of zero or lower and those following the precipitous drop accompanying detachment. Next, the actual fitting is performed by minimizing the sum of the squares of the difference between each measured point and the function to be fit using the Nelder-Mead method [30]. For the WLC case, the function minimized is,

$$
E(L, b, l)=\sum_{(x, f) \in P}\left(f_{\mathrm{WLC}}(L, b, x)-f\right)^{2},
$$

where $L$ and $b$ are model parameters, $f_{\mathrm{WLC}}$ is the force function for the WLC model described in Equation 2 at a fixed temperature, and $P$ is the set of all measurements. The Hookean case is handled in a similar manner using Equation 1 with the $k_{s}$ and $l$ parameters.

The fittings of one of our measurements to the WLC model and the Hookean model can be seen in Figure 6 respectively. The use of the more complex WLC model is justified by its much tighter fit to the experimental data.

\subsection{Measurement of Thin Fibrin Fiber Behavior}

Experiments concerning whole thin fibrin fibers (with approximately 10 protofibrils each) are performed using the same equipment as described in Section 3.1 Instead of measuring force as an individual monomer is lifted off a surface, these experiments focus on determining the breakage force of an individual fiber whose two ends have been affixed to the surface. To reduce the effects of friction, there is a gap in the surface over which the fiber is draped. The fiber is then stretched laterally, resulting in the collection of force over displacement measurements. 


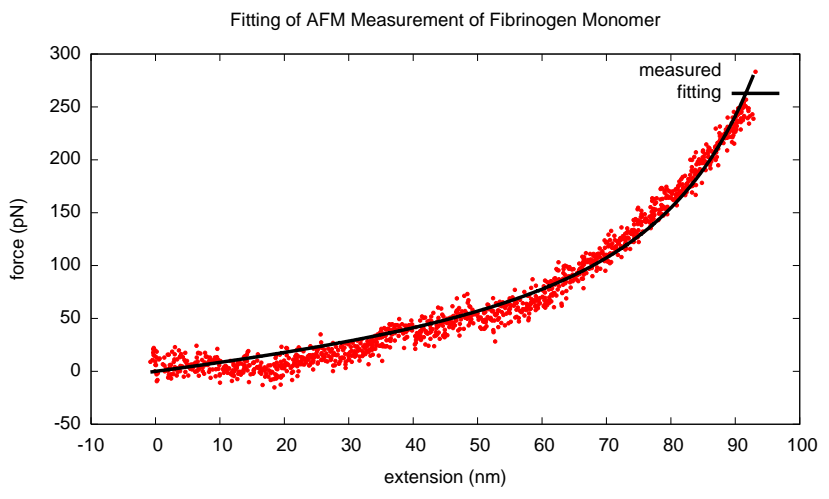

(a) WLC model fitting with parameters $L=63.0 \mathrm{~nm}$ and $b=0.059 \mathrm{~nm}$

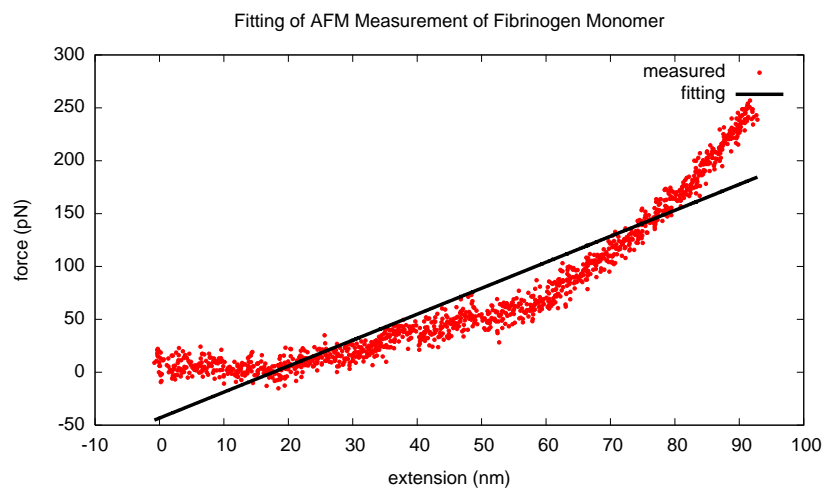

(b) Hookean model fitting with parameters $k_{s}=2.45 \mathrm{pN} / \mathrm{nm}$ and $l=$ $17.6 \mathrm{~nm}$

Figure 6: Measurement data acquired from the AFM along with its fitting to the two models. Notice how the WLC model tends to fit the data much better than a simple Hookean model.

\section{Simulation Framework}

After the experimental data has been acquired and fitted to our fibrin monomer model, the interactive simulation of the protofibril system is performed. The protofibril is represented as a particle system consisting of different classes of particles (free and static) whose interaction is dictated by specialized forces and constraints. Each free particle has a force accumulator, into which the various forces applied to the particle through interaction is summed. There are also static particles, which do not move according to Newton's laws of motion, but rather according to an externally controlled function. After all forces have been accumulated, the main equation of motion is numerically integrated using a fourth order Runge-Kutta scheme to update the positions and velocities of all free particles. Time step size as well as the exact distances between various components of the protofibril assembly are tunable parameters. For the time step size, we choose values small enough to maintain stability, but large enough to maximize interactivity. The distances between the components are estimated from measurements in the fibrin literature.

\subsection{Fibrin Monomers}

Each fibrin monomer is represented as three particles, which correspond to each of the three domains shown in Figure 4 The "coiled- coil linkers" are modeled by connecting the particle representing the central domain to those two particles representing the end domains with separate WLC or Hookean forces. The parameters from these forces are derived from the fitting performed in Section 3.3 Because each stretchable chain is only half the length of a full fibrinogen molecule, some adjustment may be necessary for the parameters. In the Hookean case, the parameters require no modification as the force produced by elongating two half-sized elements half as much is the same as the force produced by extending one full-sized element. For the WLC model, the contour length needs to be halved to compensate for the use of two half-sized elements instead of one full-sized element. Examining Equation 2, it can be seen that halving the contour length in this case ensures that the fraction $\frac{x}{L}$, which drives the increase in force, remains the same. The monomers are then replicated as many times as needed in an arrangement shown in Figure 3 to form a protofibril. A viscous drag term is added to model the overwhelming dominance of noninertial forces at the nanoscale. This has the fortunate side effect of greatly stabilizing the simulation and enabling somewhat larger time steps.

\subsection{Bonds}

In the Chemistry literature, bonds are often modeled by potential energy wells [31]. However, because the dimensions of a protofibril are orders of magnitude larger than the bond distances, both strong and weak bonds present in the protofibril can be modeled more simply. From this macroscopic perspective, as force is applied to a bond, no deformation occurs until a certain amount of force has been reached, at which point the bond instantaneously breaks. Initially we chose to represent our chemical bonds as Hookean springs, but this led to undesirable stretching. Unfortunately, as the stiffness of these springs was increased to correct for this problem, smaller time steps had to be taken to maintain stability, which limited our ability to perform the simulation interactively. To remedy this problem, constrained dynamics are employed to represent the intermonomer bonds as rigid distance constraints that break above specified force thresholds.

We briefly describe a formulation of general constraint dynamics [53]. At the core of this method is the use of constraint forces to hold certain particles in place according to various constraint functions. The primary equation for determining these constraint forces, $\hat{Q}$, is,

$$
\boldsymbol{J} \boldsymbol{W} \boldsymbol{J}^{T} \lambda=-\dot{J} \dot{\boldsymbol{q}}-\boldsymbol{J} \boldsymbol{W} \boldsymbol{Q}-k_{s} \boldsymbol{C}-k_{d} \dot{\boldsymbol{C}},
$$

where,

$$
\hat{\boldsymbol{Q}}=\boldsymbol{J}^{T} \lambda
$$

$\boldsymbol{J}$ is the Jacobian matrix for the constraint functions over force $\left(\frac{\partial \boldsymbol{C}}{\partial \boldsymbol{q}}\right)$. $\boldsymbol{W}$ is the inverted mass matrix containing a row and column for each particle. $\boldsymbol{Q}$ is the amount of other, non-constraint forces to be applied to the system. $\dot{\boldsymbol{q}}$ is the velocity of all particles. $\boldsymbol{C}$ is a vector containing the current value of each constraint function in use. The use of a dot over all quantities represents the derivative with respect to time. Strictly speaking, the last two terms on the right side are not mathematically necessary to ensure constraints are met. However, in practice these terms containing the tunable constants $k_{d}$ and $k_{s}$ combat numerical drift by elastically pulling the constraint values back to zero as necessary. Multiplication of known quantities results in a linear system with $\lambda$ as the unknown vector quantity. Because the matrices are sparse, we use a conjugate gradient method [45] is used to efficiently solve for $\lambda$ which is then multiplied by $\boldsymbol{J}^{T}$ to obtain the constraint forces on each particle. The constraint forces are then added to the total force acting on each particle.

For representing bonds, we choose a constraint function which ensures that two particles remain a specified distance from one an- 


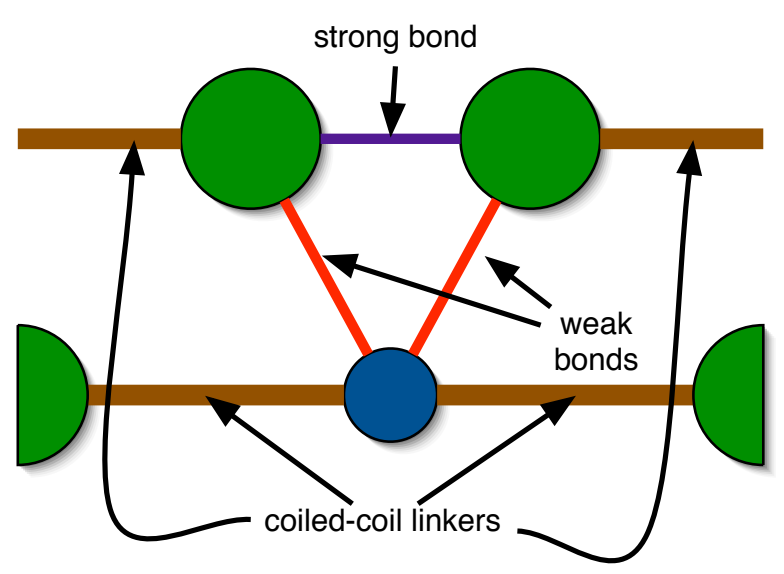

Figure 7: Simplified chemical bonding structure connecting fibrin monomers in a protofibril chain.

other so long as the bond has not been broken. Before breakage, the function is,

$$
C\left(\boldsymbol{x}_{1}, \boldsymbol{x}_{2}\right)=\left\|\boldsymbol{x}_{1}-\boldsymbol{x}_{2}\right\|^{2}-r^{2}=0,
$$

where $x_{1}$ and $x_{2}$ are 3D vector valued locations of the two constrained particles and $r$ is the distance they are to remain apart. All bonds start out intact, but will break when a certain force threshold is reached. Although these thresholds can be set to arbitrary values, they have generally been observed to differ by an approximate factor of 10. After a bond has broken, the constraint is inactivated and consequently no longer produces constraint forces on its particles. Figure 7 shows the geometric arrangement of the strong and weak bonds. The only difference between these two kinds of bonds is that weak bonds use a lower force threshold.

\subsection{Thin Fibers}

For constructing an individual thin fibrin fiber, we have implemented the model described by Yang et al. [54] in the medical literature. This structure is best shown in Figure 8 While this is one of several models proposed for how protofibrils structure into thin fibers (for example see also that of Weisel [50]), the quantitative description of the Yang model lends itself best to implementation in a computer simulation. Additional weak bonds between different protofibrils not specified in the model as described by Yang et al. are added to provide a kind of cement that holds the assembly together.

\subsection{Virtual Experiments}

Once the particles, forces and constraints have been properly set up, the virtual experiment of pulling on a protofibril or fibrin fiber begins. Mimicking laboratory conditions, one end of the structure is held in place by designating those particles as fixed. Particles on the other end are designated as special kinds of static particles (so called "mover particles") that move according to one of two possible functions. For thin fibers, empirically we have found it best to use a simple linear (constant velocity) function, while for protofibrils another method has proven to work well:

$$
\dot{x}_{p}=\left\{\begin{array}{ll}
v & \text { if } \mathrm{E}_{\mathrm{k}} \leq \varepsilon \\
0 & \text { if } \mathrm{E}_{\mathrm{k}}>\varepsilon
\end{array},\right.
$$

where $v$ is the pull velocity, $E_{k}$ is the total kinetic energy of the system $\left(\frac{1}{2} \sum_{i} \dot{x}_{i}^{2}\right)$ and $\varepsilon$ is the kinetic energy threshold. The effect of this

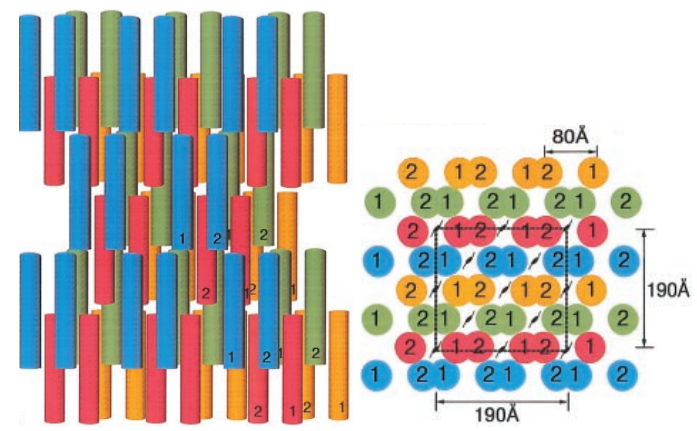

$\begin{array}{ll}\text { (a) top }(x-z) \text { view } & \text { (b) end }(x-y) \text { view }\end{array}$

Figure 8: The structure of an individual fibrin fiber from two viewpoints [54]. Each colored cylinder represents a monomer chain.

movement function is that the chain is only pulled when the kinetic energy of the system is low. This causes the effective pull speed to be low enough to avoid significantly delayed force propagation, yet high enough to be interactive. Essentially, this approximates a static simulation that quickly reaches its equilibrium through the use of a dynamic simulation.

\subsection{Visualization}

Our choice of display of the protofibril assembly is inspired by hand drawn interpretations (in 2D containing lines and circles) and physical models (in 3D consisting of spheres and ribbons) of protofibrils found in the Pathology and Biochemistry literature [12, 50]. While this is possibly a natural and simple choice for displaying the geometry of a protofibril, it has the added effect of being familiar to scientists working in the area, which are the intended end users of our system. However, we go beyond these basic visualization techniques, most importantly adding motion derived from physical simulation to show how the location of the components evolves over time. Secondary visual information concerning bond breakage and stretchable chain elongation is also provided.

End domain particles and central domain particles are both shown as spheres, with the central domain spheres having a slightly smaller radius size than the end domain spheres to correspond to their smaller size in an actual fibrin monomer [12]. The spheres are colored a blue color. The linkers that connect particles at the fibrin monomer level are shown as thin ribbons, roughly approximating their physical structure. In the WLC case, the ribbons are colored according to the fraction of the current elongation over the maximum allowed in the model (contour length) via a black body radiation color map (black to red to orange to yellow to white). In the Hookean case, because there is no implicit maximum extension length, the ribbons are given a constant red color. The bonds that connect the particles at the protofibril level are rendered as thin cylinders, which are colored according to the fraction of the current force exerted on the bond over its breakage threshold via a color map that is similar to black body radiation but which uses green instead of red (going from black to green to yellow to white).

In both cases, the color maps are clipped in order to remove white for print media display and black for on-screen display. Correspondingly, a white background is used in print and a black one in on-screen display. We explicitly avoid the use of the rainbow color map here for its lack of an intuitive connection between values and colors, uneven transitions between colors and lack of variance in luminance. The black body and green color maps do not 


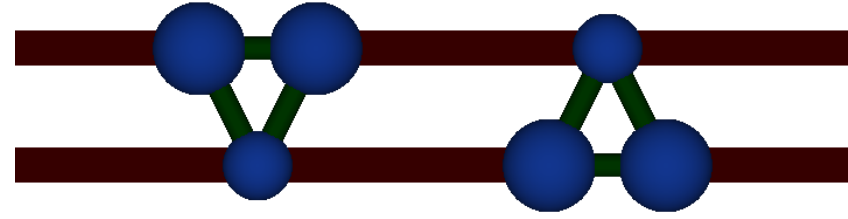

Figure 9: Zoomed-in screen shot of protofibril at rest. Notice that both the bonds and the linkers are colored darkly to show their lack of stress and extension respectively.

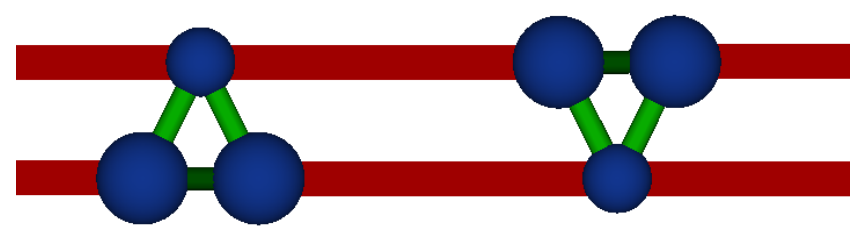

Figure 10: Here the linker is colored a brighter red, indicating that it has begun to be extended. The strong (horizontal) bonds are still relatively darkly colored, while the weak bonds (diagonal) are lighter indicating that the latter is closer to breaking.

suffer from these problems. Furthermore, the use of two different but similar color maps makes it easier for the viewer to distinguish between the bonds and coiled-coil linkers when the protofibril is in an unstressed state. As each set of components nears its maximum sustainable force, the color maps converge to yellow in print or white in display. However, because at this point the viewer has presumably already located the components they are interested in observing, the lack of differing color is not a problem. This coloring of the linkages between particles enables the viewer to better see the particular state of the entire model. In particular, this information is most important in the case of bond breakage, since the current stress placed on the bond is not visible in any other way.

\section{Results}

We have implemented the data fitting method described in Section 3.3 as an offline preprocess and the simulation method described in Section 4 as an interactive tool.

\subsection{Implementation Details}

The fitting preprocess is performed using a set of tools developed in Python [33] that make use of the third party software, SciPy [41] and Numeric [2] modules. The interactive simulation and visualization tool contains several pieces. The simulation engine was developed as an efficient Objective-C library that makes use of optimized BLAS [9] routines for improved performance. The actual tools have been built using the Cocoa framework on the Apple Macintosh platform and GNUstep on Linux.

\subsection{Protofibril Simulation}

Figures 9 through 13 show simulated pulling of a protofibril going from a state of rest through increasing tension and finally past the stage of bond breakage. For these images, the strong horizontal bonds' breaking threshold was set to $100 \mathrm{pN}$, while for the weak vertical bonds it was set to $10 \mathrm{pN}$. Although in the actual system a black background is used, a white one has been used to here for better display on paper. The color maps have been clamped in a manner so as not to use colors too close to white. In addition to the visualization that helps the user understand the behavior

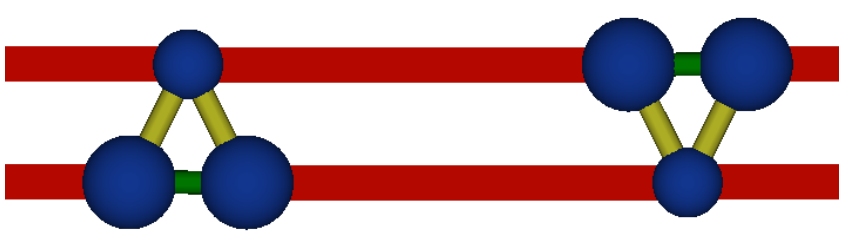

Figure 11: Both bonds and linkers have become brighter as the pulling of the molecule continues, showing the increasing stress and extension.

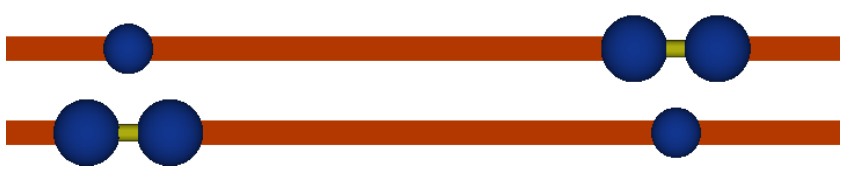

Figure 12: The weak bonds have now broken, as demonstrated by their absence from the image. The strong bonds are now quite bright, indicating that they are close to breaking. Similarly, the linkers are also brighter showing their continued extension.

of the protofibril, we have also extracted the force profile of the simulated pulling of the protofibril using the WLC model (Figure 14). For these simulations, different breaking thresholds were used $(50 / 500 \mathrm{pN})$.

\subsection{Validation of Thin Fiber Simulation}

Figure 15(a) shows the experimentally measured force profile of a typical thin fiber, while Figure 15(b) shows our simulation of a similar fiber. Note that the behavior is largely similar, although not identical due to simplifications in the underlying fiber model. Parameters for the monomers acquired earlier have been used here as well, while non-measured parameters and distances used come from medical fibrin literature discussed earlier in Section 1 and 2

\subsection{Performance}

On a $1.33 \mathrm{GHz}$ G4 Apple Powerbook with $768 \mathrm{MB}$ of RAM, we are able to simulate a typically sized individual fiber containing approximately 15 protofibrils $(5 \times 5 \times 1$ grid with the requisite gaps described in Figure 8) at nearly 20 frames per second. Profiling of the running time indicate that for smaller chains, graphical display is the limiting performance factor. However, as the number of monomers in the chain is increased, the simulation time quickly becomes the dominant factor. Specifically, the performance of the linear algebra routines takes up the largest amount of program run time for larger systems with more bonds.

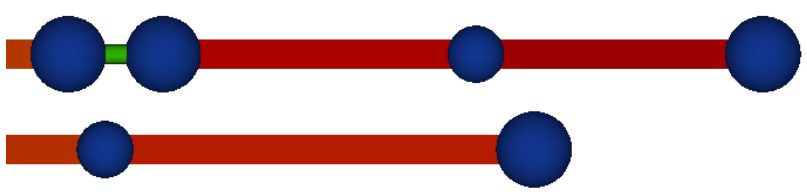

Figure 13: Some strong bonds have now broken, causing the molecular chain to split into disjoint parts. A portion detached on its right end is shown. Notice how the color of the linkers and the remaining strong bonds have returned to a darker color as the elongation and stress have decreased. 


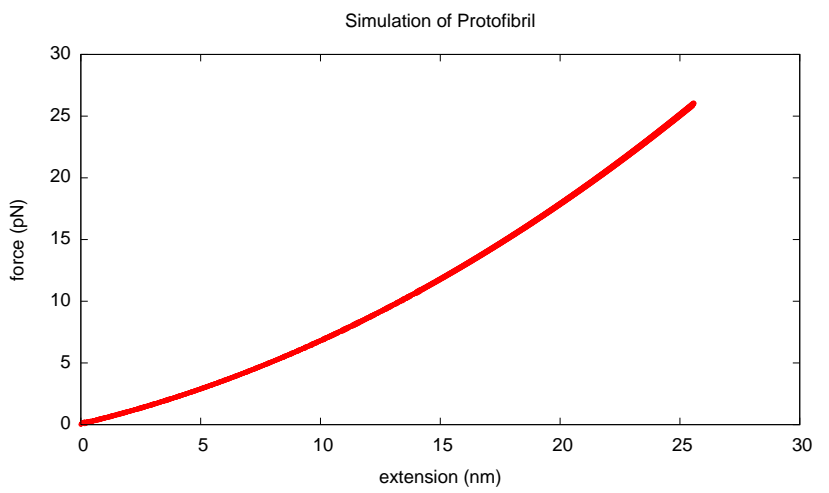

Figure 14: Force profile of the simulation of a single protofibril using the WLC model.

\subsection{Discussion and Analysis}

Although we have made simplifying assumptions primarily by representing a complex molecule consisting of tens of thousands of atoms as a kind of exponential spring, qualitative comparisons between actual experimental data and our simulation results indicate the adequacy and appropriateness of our assumptions. More importantly, the extreme complexity of many biological systems necessitates such dramatic simplifications for the simulations to be computationally tractable. At the same time, even with these simplifications, the intuitive meaning of many of the remaining parameters of the complex system often is unclear. Fortunately, as we have shown here, experimental data can be used in an automatic process for determining optimal values of these parameters.

Furthermore, measurements taken from actual real-world data at one level of hierarchy can be used as a building block for modeling other levels in a complex hierarchical system. Such an approach can be valuable when information or data at the other levels may not be available for a number of reasons, such as technological or experimental limitations or missing data.

\section{CONCLUSion ANd Future Work}

In this paper, we have presented a novel method for interactively simulating and visualizing fibrin fibers, based on measurements acquired using an AFM via the nanoManipulator. Traditionally laboratory experiments have been carried out to derive fundamental laws and a basic understanding of highly complex biological systems. We suggest that a combination of data-driven modeling based on experimental measurements and physically-based simulation built upon the empirical models at the lower levels of a hierarchical system can potentially provide a powerful tool in a "virtual laboratory" to assist and possibly accelerate scientific discovery.

One of our long-term goals is to incorporate larger scale layers into a unified multiscale physically-based, data-driven model of fibrin fibers and fibrin webs. Additionally, there is the possibility of investigating the application of such approaches to interactive manipulation of other nanoscale structures and materials, such as the system of cilia in the lungs, in a virtual environment.

\section{REFERENCES}

[1] Brett Allen, Brian Curless, and Zoran Popovic. Articulated body deformation from range scan data. In Proceedings of SIGGRAPH 2002, pages 612-619, New York, NY, USA, 2002. ACM Press.

[2] David Ascher, Paul F. Dubouis, Konrad Hinsen, Jim Hugunin, and Travis Oliphant. Numerical python users' guide. Technical Report UCRL-MA-128569, Lawrence Livermore National Laboratory, 2001.

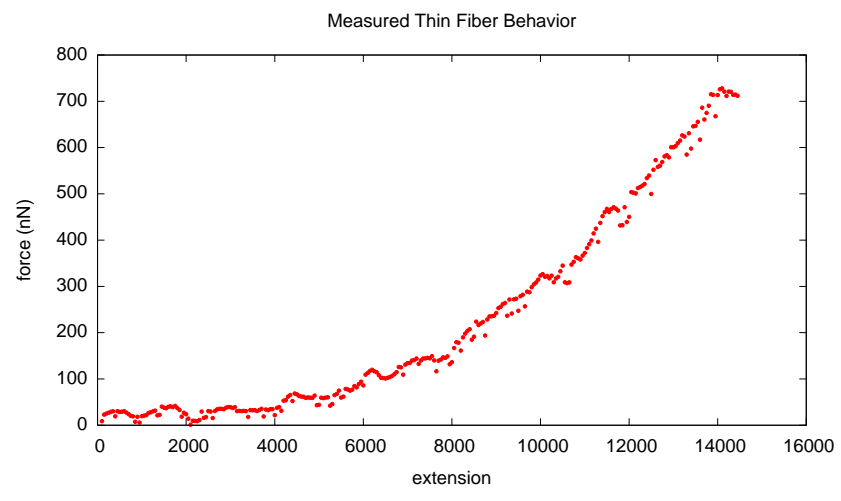

(a) measured

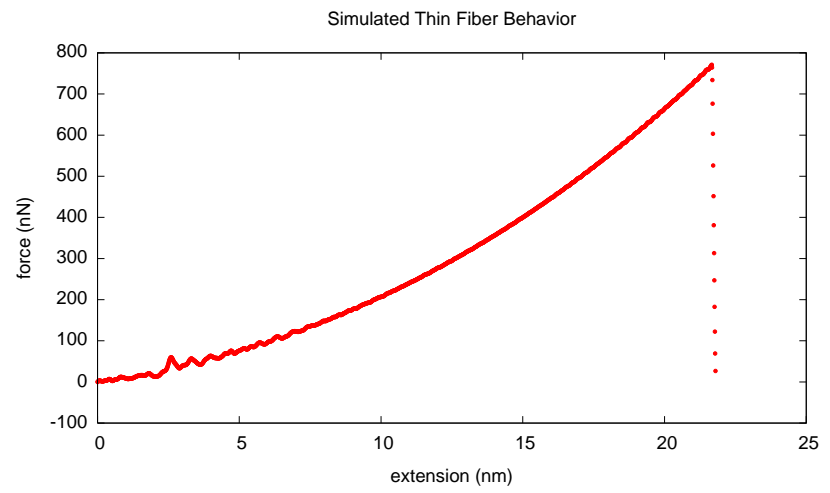

(b) simulated

Figure 15: Validation of simulation results: (a) Measured vs. (b) simulated force profiles for a thin fiber.

[3] G. Binnig, C.F. Quate, and Ch. Gerber. Atomic force microscope. Physical Review Letters, 56(9):930-933, 1986.

[4] W. Bouma, X. Chen, I. Fudos, C. Hoffmann, and P. Vermeer. An Electronic Primer on Geometric Constraint Solving. http://www.cs.purdue.edu/homes/cmh/electrobook/intro.html, 1990.

[5] Iman Brouwer, Jeffrey Ustin, Loren Bentley, Alana Sherman, Neel Dhruv, and Frank Tendick. Measuring in vivo animal soft tissue properties for haptic modeling in surgical simulation. Medicine Meets Virtual Reality, pages 69-74, 2001.

[6] B. Bruderlin and D. Roller (eds). Geometric Constraint Solving and Applications. Spring Verlag, 1998.

[7] D. d'Aulignac, M.C. Cavusoglu, and C. Laugier. Modeling the human thigh for a realistic echographic simulator with force feedback. In Proceedings of the International Conference on Medical Image Computer-Assisted Intervention, pages 1191-1198, Cambridge, United Kingdom, September 1999.

[8] Paul E. Debevec, Camillo J. Taylor, and Jitendra Malik. Modeling and rendering architecture from photographs: a hybrid geometry- and image-based approach. In Proceedings of SIGGRAPH 1996, pages 11-20, New York, NY, USA, 1996. ACM Press.

[9] Jack J. Dongarra, Jeremy Du Croz, Sven Hammarling, and Iain Duff. Algorithm 679: A set of level 3 Basic Linear Algebra Subprograms: Model implementation and test programs. ACM Transactions on Mathematical Software, 16(1):18-28, March 1990.

[10] R. F. Doolittle. Structural basis of the fibrinogen-fibrin transformation. Blood Reviews, 17:33-41, 2003.

[11] J. D. Ferry, M. Miller, S. Shulman, and Arch. Biochem. The conver- 
sion of fibrinogen to fibrin. Biophysics, 34:424-436, 1951.

[12] W. E. Fowler, R. R. Hantgan, J. Hermans, and H. P. Erickson. Structure of the fibrin protofibril. Proceedings of the National Academy of Sciences of the USA, 78(8):4872-4876, August 1981.

[13] C. Gerth, W. W. Roberts, and J. D. Ferry. Rheology of fibrin clots. Biophysical Chemistry, 2:208-217, 1974.

[14] C. J. Glover, L. V. McIntire, C. H. Brown, and E. A. Natelson. Rheological properties of fibrin clots - effects of fibrinogen concentration, factor-8 deficiency, and factor-13 inhibition. Journal of Laboratory and Clinical Medicine, 86:644-656, 1975.

[15] M. Guthold, W. Liu, B. Stephens, S. T. Lord, R. R. Hantgan, D. A. Erie, Jr. R. M. Taylor, and R. Superfine. Visualization and mechanical manipulations of individual fibrin fibers suggest that fiber cross section has fractal dimension 1.3. Biophysical Journal, 87:4226-4236, 2004.

[16] C. E. Hall and H. S. Slayter. J. Biophys. Biochem. Cytol., pages 11-16, 1959.

[17] M. Hauth, J. Groß, and W. Straßer. Interactive physically based solid dynamics. In Proceedings of the ACM SIGGRAPH/Eurographics Symposium on Computer Animation, pages 17-27. Eurographics Association, 2003.

[18] R. M. Taylor II, D. Borland, Jr. F. P. Brooks, M. Falvo, M. Guthold, T. Hudson, K. Jeffay, G. Jones, D. Marshburn, S. J. Papadakis, L.C. Qin, A. Seeger, F. D. Smith, D. H. Sonnenwald, R. Superfine S. Washburn, C. Weigle, M. C. Whitton, P. Williams, L. Vicci, and W. Robinett. Visualization Handbook, chapter Visualization and Natural Control Systems for Microscopy, pages 875-900. Harcourt Academic Press, 2004.

[19] P. A. Janmey, E. J. Amis, and J. D. Ferry. Viscoelastic properties of fibrin clots in large shearing deformations. Journal of Rheology, 26:599-600, 1982.

[20] P. A. Janmey, E. J. Amis, and J. D. Ferry. Rheology of fibrin clots .6. stress-relaxation, creep, and differential dynamic modulus of fine clots in large shearing deformation. Journal of Rheology, 27:135-153, 1983.

[21] G. Kramer. Solving Geometric Constraint Systems: A case study in kinematics. MIT Press, 1992.

[22] Jochen Lang, Dinesh K. Pai, and Hans-Peter Seidel. Scanning largescale articulated deformations. In Graphics Interface, pages 265-272, June 2003.

[23] Jochen Lang, Dinesh K. Pai, and Robert J. Woodham. Acquisition of elastic models for interactive simulation. The International Journal of Robotics Research, 21(8):713-733, August 2002.

[24] Hendrik P. A. Lensch, Jan Kautz, Michael Goesele, Wolfgang Heidrich, and Hans-Peter Seidel. Image-based reconstruction of spatial appearance and geometric detail. ACM Trans. Graph., 22(2):234-257, 2003.

[25] H. Maaß and U. Kühnapfel. Noninvasive measurement of elastic properties of living tissue. In 13th Int. Congress on Comp. Assisted Radiology (CARS), pages 865-870, 1999.

[26] Wojciech Matusik, Hanspeter Pfister, Matt Brand, and Leonard McMillan. A data-driven reflectance model. ACM Trans. Graph. 22(3):759-769, 2003.

[27] L. F. Mockros, W. W. Roberts, and L. Lorand. Viscoelastic properties of ligation-inhibited fibrin clots. Biophysical Chemistry, 2:164-169, 1974

[28] G. W. Nelb, C. Gerth, J. D. Ferry, and L. Lorand. Rheology of fibrin clots .3. shear creep and creep recovery of fine ligated and coarse unligated clots. Biophysical Chemistry, 5(3):377-387, 1976.

[29] G. W. Nelb, G. W. Kamykowski, and J. D. Ferry. Rheology of fibrin clots .5. shear modulus, creep, and creep recovery of fine unligated clots. Biophysical Chemistry, 13(1):15-23, 1981.

[30] J.A. Nelder and R. Mead. A simplex method for function minimization. Computer Journal, 7:308-313, 1965.

[31] David W. Oxtoby and Norman H. Nachtrieb. Principles of Modern Chemistry. Saunders College Publishing, Fort Worth, third edition, 1996.

[32] Dinesh K. Pai, Kees van den Doel, Doug L. James, Jochen Lang, John E. Lloyd, Joshua L. Richmond, and Som H. Yau. Scanning physical interaction behavior of $3 \mathrm{~d}$ objects. In Proceedings of SIGGRAPH, pages 87-96. ACM Press, 2001.

[33] Python 2.3.3. http://www.python.org

[34] Matthias Rief, Julio M. Fernandez, and Hermann E. Gaub. Elastically coupled two-level systems as a model for biopolymer extensibility. Physical Review Letters, 81(21):4764-4767, 1998.

[35] Matthias Rief, Mathias Gautel, Filipp Oesterhelt, Julio M. Fernandez, and Hermann E. Gaub. Reversible unfolding of individual titin immunoglobulin domains by afm. Science, 276(5315):1109-1112, May 1997.

[36] W. W. Roberts, L. L. Lorand, and L. F. Mockros. Viscoelastic properties of fibrin clots. Biorheology, 10:29-42, 1973.

[37] Szymon Rusinkiewicz, Olaf Hall-Holt, and Marc Levoy. Real-time 3d model acquisition. In Proceedings of SIGGRAPH 2002, pages 438446, New York, NY, USA, 2002. ACM Press.

[38] E. A. Ryan, L. F. Mockros, A. M. Stern, and L. Lorand. Structural origins of fibrin clot rheology. Biophysical Journal, 77:2827-2836, 1999.

[39] E. A. Ryan, L. F. Mockros, J. W. Weisel, and L. Lorand. Influence of a natural and a synthetic inhibitor of factor xiiia on fibrin clot rheology. Biophysical Journal, 77:2813-2826, 1999.

[40] Jeffrey L. Schoner, Jochen Lang, and Hans-Peter Seidel Measurement-based interactive simulation of viscoelastic solids. Computer Graphics Forum, 23(3):547-556, 2004.

[41] Scipy 0.3.2. http://www.scipy.org

[42] M. C. Scrutton, S. B. Rossmurphy, G. M. Bennett, Y. Stirling, and T. W. Meade. Changes in clot deformability - a possible explanation for the epidemiologic association between plasma-fibrinogen concentration and myocardial-infarction. Blood Coagulation \& Fibrinolysis, 5(5):719-723, 1994

[43] L. Shen and L. Lorand. Contribution of fibrin stabilization to clot strength - supplementation of factor-xiii-deficient plasma with the purified zymogen. Journal of Clinical Investigation, 71:1336-1341, 1983.

[44] L. L. Shen, J. Hermans, J. McDonagh, R. P. McDonagh, and M. Carr Effects of calcium-ion and covalent crosslinking on formation and elasticity of fibrin gels. Thrombosis Research, 6:255-265, 1975.

[45] Jonathan Shewchuck. An introduction to the conjugate gradient method without the agonizing pain. http://www.cs.cmu.edu/ quake-papers/painless-conjugate-gradient.pdf August 1994.

[46] Matthew Stone, Doug DeCarlo, Insuk Oh, Christian Rodriguez, Adrian Stere, Alyssa Lees, and Chris Bregler. Speaking with hands: creating animated conversational characters from recordings of human performance. ACM Trans. Graph., 23(3):506-513, 2004.

[47] Russell M. Taylor, Warren Robinett, Vernon L. Chi, Jr. Frederick P. Brooks, William V. Wright, R. Stanley Williams, and Erik J. Snyder. The nanomanipulator: a virtual-reality interface for a scanning tunneling microscope. In Proceedings of SIGGRAPH 1993, pages 127-134, New York, NY, USA, 1993. ACM Press.

[48] W. A. Voter, C. Lucaveche, and H. P. Erickson. Concentration of protein in fibrin fibers and fibrinogen polymers determined by refractiveindex matching. Biopolymers, 25(Dec):2375-2373, 1986.

[49] V. Vuskovic, M. Kauer, G. Székely, and M. Reidy. Realistic force feedback for virtual reality based diagnostic surgery simulators. In Proceedings of the IEEE International Conference on Robotics and Automation, pages 1592-1598, San Francisco, 2000.

[50] John W. Weisel, Chandrasekar Nagaswami, and Lee Makowski. Twisting of fibrin fibers limits their radial growth. Proceedings of the National Academy of Sciences of the USA, 84:8991-8995, December 1987.

[51] J.W. Weisel. Fibrinogen and fibrin. Advanced Protein Chemistry, 70:247-299, 2005

[52] R. C. Williams. Morphology of bovine fibrinogen monomers and fibrin oligomers. Journal of Molecular Biology, 150:399-408, 1981

[53] Andrew Witkin. SIGGRAPH 2001 Course Notes: Physically Based Modeling, chapter 'Constrained Dynamics'.

[54] Zhe Yang, Igor Mochalkin, and Russell F. Doolittle. A model of fibrin formation based on crystal structures of fibrinogen and fibrin fragments complexed with synthetic peptides. Proceedings of the National Academy of Sciences of the USA, 97:14156-14161, 2000. 